\title{
Mauriac en une, Mauriac en livre, Mauriac en ligne. Réflexion sur les dispositifs éditoriaux
}

Mauriac, From Newspapers to the Web. Reflection on Editorial Devices

\section{Philippe Baudorre et Jessica de Bideran}

\section{(2) OpenEdition}

\section{Journals}

Édition électronique

URL : https://journals.openedition.org/revuehn/325

DOI : $10.4000 /$ revuehn. 325

ISSN : 2736-2337

Éditeur

Humanistica

\section{Référence électronique}

Philippe Baudorre et Jessica de Bideran, « Mauriac en une, Mauriac en livre, Mauriac en ligne. Réflexion sur les dispositifs éditoriaux », Humanités numériques [En ligne], 1 | 2020, mis en ligne le 01 janvier 2020, consulté le 17 juillet 2021. URL : http://journals.openedition.org/revuehn/325 ; DOI https://doi.org/10.4000/revuehn.325

Les contenus de la revue Humanités numériques sont mis à disposition selon les termes de la Licence Creative Commons Attribution 4.0 International. 


\title{
humanités \\ numériques
}

\section{Mauriac en une, Mauriac en livre, Mauriac en ligne. Réflexion sur les dispositifs éditoriaux}

\section{Mauriac, From Newspapers to the Web. Reflection on Editorial Devices}

\author{
Philippe Baudorre et Jessica de Bideran
}

\section{Résumés}

À l'ère où fabrication et diffusion des textes convergent dans un même environnement numérique, ce texte se propose de revenir, à partir d'un exemple précis, sur les contextes de lecture que nous élaborons progressivement à travers nos diverses politiques de publicisation en ligne. Les " gestes " que nous mettons en œuvre s'inscrivent en effet dans une réflexion plus générale sur les migrations textuelles et, plus particulièrement, sur le déplacement de productions "de presse ", du média pour lequel elles ont été conçues vers d'autres médias qui vont les recueillir, le livre d'abord, le support numérique ensuite.

In an era when writing and reading converge in the same digital environment, we would like to think about the digitisation of the literary heritage and about the reading contexts that we are building. This paper proposes a reflection on textual migrations and more particularly on the displacement of "press" productions to another, digital medium.

\section{Entrées d'index}

MOTS-CLÉS : littérature, analyse des usages, corpus d'auteur, dispositif éditorial, édition électronique, éditorialisation, numérisation, remédiatisation

KEYWORDS: literature, usage analysis, authorial corpus, editorial system, digital editing, editorialisation, digitisation, remediation 
Tout outil, et a fortiori tout dispositif ${ }^{1}$ technique plus complexe, inclut et préfigure, dans sa conception même et son évolution, un ou des " usages ». Ces usages, réguliers ou ponctuels, quelle que soit leur orientation - professionnelle ou ludique, manuelle ou intellectuelle, immédiate ou inscrite dans une activité plus longue, récurrente ou non -, supposent un objectif clairement identifié, une maîtrise de l'outil et une performance plus ou moins évaluable. Ils peuvent s'intégrer dans des "pratiques " plus complexes qui impliquent méthode, apprentissage, normes, partage collectif, reconnaissance sociale et, donc, une extension dans le temps individuel ou collectif (évolution des pratiques, réajustements, progrès) ainsi qu'au sein de l'espace social (succès, engouement, mode ou obsolescence).

Si les pratiques instrumentées et les techniques d'expérimentation inhérentes sont largement débattues au sein de la communauté des humanités numériques (de la transcription à la lecture distante en passant par la fouille de données), la question des dispositifs créés pour rendre publics ces travaux par les équipes engagées dans ces programmes reste encore à la marge. Or, parce qu'elles participent à inventer et à construire de nouvelles formes d'“ édition ouverte " (Le Deuff 2016), ces équipes participent aussi à l'imposition de nouvelles formes d'écrits d'écrans qui encadrent, comme le rappellent Emmanuel Souchier et Yves Jeanneret (2005), les circulations et les réceptions de ces objets textuels.

À l'ère où fabrication et diffusion des textes convergent dans un même environnement numérique (Buard 2015), nous souhaitons réfléchir ici aux contextes de lecture que nous élaborons progressivement à travers nos diverses politiques de publicisation. Sans doute, l'objet sur lequel nous travaillons, c'est-à-dire un corpus d'articles de presse, et le contexte dans lequel nous portons ce projet, à savoir un programme de recherche financé par la région Nouvelle-Aquitaine, impriment-ils des questionnements et des postures spécifiques à notre équipe. Mais nous confrontons ces questionnements aux activités et projets des membres du consortium CAHIER ${ }^{2}$, auquel nous sommes associés depuis 2015. Bien que ce texte prenne essentiellement appui sur notre propre expérimentation, il nous semble donc pouvoir poser quelques problématiques qui traversent les humanités numériques.

Les « gestes » que nous mettons en œuvre (numérisation, transcription, indexation, exposition, etc.) s'inscrivent en effet dans une réflexion plus générale sur les migrations textuelles et plus particulièrement sur le déplacement de productions "de presse ", du média pour lequel elles ont été conçues vers d'autres médias qui vont les recueillir, le livre d'abord, le support numérique ensuite. Notre corpus est certes circonscrit et clairement délimité, mais il s'inscrit aussi dans une histoire longue de ces migrations, histoire que l'on peut faire remonter à la première moitié $\mathrm{du} \mathrm{XIX}^{\mathrm{e}}$ pour la première partie (du journal vers le livre), histoire plus récente pour la deuxième étape (vers le numérique). Sans être dans une confrontation binaire de l'imprimé enfermant et du numérique délivrant, notre présupposé est que cette deuxième vague migratoire réactualise et en même temps déplace des questions que la première posait déjà. L'observation participante que nous explorons ici, dans un exercice d'auto-ethnographie qui nous amène à décrire les gestes et pratiques que nous forgeons progressivement dans le cadre de ce programme de recherche-action ${ }^{3}$, nous permet in fine de mettre en 
évidence ce qui se passe dans la "communication littéraire ", c'est-à-dire dans l'interaction entre producteur, énoncé et récepteur lorsque ces éléments restent relativement stables (ils ne peuvent le rester totalement, mais ce n'est pas notre propos ici) et que la variante principale s'établit au niveau du support ou du canal de médiatisation ou médiation ${ }^{4}$.

Après avoir rappelé le contexte théorique et historique qui est le nôtre, nous proposerons de confronter celui-ci à notre propre programme de recherche-action Mauriac en ligne, afin de mieux saisir « ce que le terrain fait aux concepts » (Le Marec 2002) et inversement.

\section{Des dispositifs éditoriaux comme contextes d'usage}

Entre simple " utilisation ", " usage " et " pratique ", on peut voir comme un " emboîtement " (le premier terme pouvant entrer dans le deuxième qui peut lui-même...), et une progression dans l'autonomie de l'utilisateur qui avance vers une appropriation de plus en plus complète $\mathrm{du}$ « dispositif ». Que désigne cette dernière notion?

Polysémique, cette notion complexe ne cesse d'être interrogée par les sciences de l'information et de la communication, la sociologie ou certains courants des études littéraires qui s'intéressent aux diverses formes de la circulation des savoirs. Loin de nous, toutefois, l'idée de la redéfinir ici ; disons simplement que le dispositif dont nous parlons ici est un dispositif éditorial avec ce qu'il suppose de technique (support matériel), qui est intégré à une économie qui le porte et qui souvent le précède (le livre, la presse, le numérique), et qui, enfin, se voit soumis à de multiples appropriations et symboliques selon les usages et pratiques sociales, intellectuelles ou culturelles qui se développent autour. Ces dispositifs éditoriaux résultent de ce que la sociologie du littéraire appelle l'« énonciation éditoriale ", c'est-à-dire d'une série d'actions polyphoniques qui permettent de formaliser, transformer, faire circuler l'objet textuel, mais qui restent le plus souvent invisibles aux yeux des récepteurs (Genêt 2019). En d'autres termes, le dispositif éditorial est pensé ici comme " un artefact, certes, dont le substrat est technique, mais qui dans sa matérialité même engage des rapports de communication et suppose des substances d'expression " (Jeanneret 2007) ; il cadre et sert de cadre, en définitive, à une communication médiatisée.

Numériser des corpus de textes, c'est donc certes structurer de l'information, mais c'est aussi réfléchir aux futurs espaces médiatiques qui permettront non seulement de redocumentariser ces corpus (Pédauque et Lund 2007) mais encore de faciliter leur circulation et, en fin de compte, leur réception. Autrement dit, tout chercheur s'engageant dans un projet d'édition numérique imagine et construit de nouveaux modèles de production, de publicisation et de réception des objets textuels (Eberle-Sinatra et Vitali Rosati 2014, 49). Comme l'a déjà souligné Julia Bonaccorsi (2015) au sujet des multiples présences en ligne des manuscrits de Stendhal, la base de données et le site Web deviennent, dans ce contexte, des lieux de médiation qui donnent à voir et à interpréter des collections patrimoniales et, conséquemment, font varier les qualités et réceptions mêmes de ces objets textuels en fonction d'univers organisationnels, institutionnels et scientifiques pluriels. 
Compte tenu de ces multiples possibilités, il est dès lors logique de constater qu'à l'intérieur même du consortium CAHIER, il n'existe pas de schèmes organisateurs des dispositifs de diffusion des corpus étudiés ${ }^{5}$, mais qu'un bricolage bon teint, caractéristique de l'expérimentation des humanités numériques (Boulaire et Carabelli 2017), aboutit à des espaces médiatiques bien différents (Idmhand, Riffard, et Walter 2017). Alors que le cadre de fonctionnement du numérique au sein du consortium paraît stable, souvent décrit à partir d'un processus allant de la numérisation à l'édition en passant par la structuration, les cadres d'usage demeurent, eux, pluriels.

$\mathrm{Au}$-delà toutefois de ces divergences, les projets réunis au sein du consortium CAHIER supposent tous une production textuelle bien identifiée et bien délimitée (nous traitons des " corpus d'auteurs ») et, dans la plupart des cas, une communication nativement publique de ces textes (et donc un premier dispositif de production, de publicisation et de réception). Numériser consiste alors à créer des substituts numériques d'objets textuels déjà pris dans des dispositifs éditoriaux premiers (qui peuvent être des papiers isolés, mais qui sont le plus souvent liés en cahiers, brochures ou livres) afin d'en extraire le texte dont ils étaient les supports (dans le meilleur des cas par OCR, mais aussi le plus souvent par une transcription, en TEI par exemple) et d'assurer la migration de ces textes vers un autre dispositif éditorial. Les corpus d'auteurs traités par les différents membres du consortium nous permettent ainsi de remarquer que les objets textuels sur lesquels nous travaillons relèvent initialement de deux espaces (et donc de deux formes d'usages) distincts : espace et usage privés ou espace et usage publics. Le tableau cidessous propose de les synthétiser (tableau 1).

TABLEAU 1. MODÉlISATION DES ESPACES ET CADRES D'USAGeS DES CORPUS D’AUTEURS TRAITÉS PAR LES MEMBRES DU CONSORTIUM CAHIER

\begin{tabular}{|c|c|}
\hline USAGES PRIVÉS & USAGES PUBLICS \\
\hline $\begin{array}{l}\text { Usages individuels (pour } \\
\text { soi) : } \\
\text { - les carnets consignant re- } \\
\text { marques personnelles, pen- } \\
\text { sées et journaux intimes (Les } \\
\text { Écrits de l'abbé Castel de Saint- } \\
\text { Pierre : https://www.uni- } \\
\text { caen.fr/puc/sources/castel/) } \\
\text { - les dossiers de préparation } \\
\text { (Les Dossiers de Bouvard et Pé- } \\
\text { cuchet }: \text { http://www.dossiers- } \\
\text { flaubert.fr/) } \\
\text { - les testaments (Testaments } \\
\text { de poilus : https://testaments- } \\
\text { de-poilus.huma-num.fr/) } \\
\text { Usages pluriels (partagés avec } \\
\text { au moins un autre, ou plus) : } \\
\text { - la correspondance (Corres- } \\
\text { pondance de Flaubert : } \\
\text { http://flaubert.univ-rouen.fr/ } \\
\text { correspondance/) }\end{array}$ & $\begin{array}{l}\text { - Le cours (ou la conférence) destiné à être dit et qui } \\
\text { intègre un dispositif complexe, de type pédagogique } \\
\text { (Archive numérique Desanti : http://archive.desanti.hu- } \\
\text { ma-num.fr/). } \\
\text { - Le livret d'opéra (ou le texte de théâtre) conçu en vue } \\
\text { de la représentation et qui s'intègre lui aussi dans un } \\
\text { dispositif complexe, celui de la représentation scénique } \\
\text { (Création de corpus de livrets d'opéra sous l'Ancien Régime, } \\
\text { l'Académie royale de musique de Paris : http://fanum- } \\
\text { txm.univ-fcomte.fr/txm/). } \\
\text { - Les textes de presse, destinés à une lecture en prin- } \\
\text { cipe individuelle et intégrant le dispositif complexe de } \\
\text { la presse (et son évolution, avec la presse moderne et sa } \\
\text { "matrice ": écriture collective, rubricité, périodicité, } \\
\text { cf. Thérenty 2o13) (Journaux d'Alexandre Dumas : } \\
\text { http://alexandredumas.org/). } \\
\text { - Le livre et le dispositif de la librairie ou de l'édition } \\
\text { moderne (avec de nombreuses formes, dictionnaire, } \\
\text { atlas, les collections et les genres, etc.) (Bibliothèques } \\
\text { virtuelles humanistes : http://www.bvh.univ-tours.fr/). }\end{array}$ \\
\hline
\end{tabular}

Bien sûr, les frontières entre les catégories ainsi définies sont poreuses ; la lettre peut devenir "publique » ou " ouverte ", le texte destiné à la représentation théâtrale se lire, une série d'articles devenir livre, etc. Cela conduit à distinguer des " usages natifs ", ou primaires, et des 
usages "détournés ", ou secondaires. La numérisation (et donc la mise en œuvre d'un nouveau dispositif éditorial de transcription, de transmission et de lecture) peut intervenir immédiatement après l'usage premier ou, ce qui est le plus fréquent, prendre sa place dans une longue et complexe généalogie d'usages (Jouët 2000), très éloignée dans le temps de la conception originale...

Or, parce que se lancer dans un projet de numérisation est long et coûteux, il nous semble plus que pertinent de dépasser l'effet de mode pour s'interroger sur les fondements de cette injonction contemporaine en replaçant celle-ci dans le temps long. Revenir sur l'évolution de ces contextes d'usage et sur les différentes mutations qu'ont connues ou pu connaître les objets textuels nous offre justement cette distanciation nécessaire. Nous souscrivons en ce sens totalement aux propos d'Yves Jeanneret qui souligne combien "l'usage est un processus complexe et incertain, qui suppose, pour se constituer, des retours sur l'histoire des objets et des attitudes ", une " mémoire sociale ", en somme, des productions culturelles et des stratégies de communication et de réception (Jeanneret 2007, 13).

\section{L'évolution des contextes d'usage à travers la migration des objets textuels}

Rappelons donc pour commencer que les objets textuels sur lesquels nous travaillons ont tous été détournés une première fois de leurs usages "natifs ", dans la mesure où ils ont été conservés. Le geste d'archivage, qui participe au processus de patrimonialisation (Davallon 2006) et permet à ces objets textuels de parvenir jusqu'à nous, est donc le premier détournement. Or, cette première extension du contexte d'usage transforme l'objet textuel en un document (historique, littéraire, archivistique, etc.), c'est-à-dire en une trace, une mémoire de l'activité pour laquelle il a été conçu. La lettre conservée par son destinataire (ou recopiée par son expéditeur), le brouillon simplement conservé ou classé par l'auteur, l'article de presse découpé et conservé dans un classeur s'insèrent désormais dans un dispositif de conservation qui relève d'un détournement et d'une pratique, individuelle ou publique, spontanée ou réfléchie, qui suppose un décideur, un opérateur, des normes, etc. Citons à titre d'exemple le premier legs, constitué de manuscrits autographes et dactylographiés, d'épreuves corrigées, de correspondances et de papiers personnels, effectué par François Mauriac lui-même en 1968 à la bibliothèque littéraire Jacques Doucet ${ }^{6}$ et qui atteste de son désir de transmettre à la postérité les traces de son activité créatrice, mais aussi de ses divers engagements politiques déjà valorisés par ailleurs à travers les reprises en volumes de son Journal ainsi que de ses Mémoires (nous y reviendrons). Papiers personnels, correspondance et photographies seront pour leur part conservés par la famille après le décès de l'écrivain, puis transmis quelques années plus tard à cette même institution parisienne, mais aussi à des structures régionales, rappelant ainsi la dimension girondine de cet autre patrimoine littéraire (Centre François Mauriac de Malagar et bibliothèque municipale de Bordeaux). 
L'archivage et son pendant, la conservation, constituent donc les pratiques les plus courantes et les plus systématiques de détournement des usages " natifs ». Soigneusement conservé dans un fonds, l'objet textuel est le même, mais il est aussi devenu autre ; perdant sa valeur d'usage originelle, il s'inscrit désormais dans un nouveau dispositif (le fonds d'archives ou de bibliothèque et ses procédures) et se prête à de nouveaux usages (l'étude historique par exemple). Autre détournement traditionnel, la publication sous forme de livre d'une correspondance, de journaux intimes ou d'articles de presse, qui repose sur une évolution du processus et du dispositif éditorial et qui s'intègre, pour de nombreux corpus, dans une pratique très codifiée, celle de l'édition critique ${ }^{7}$.

Cette mutation d'une pratique individuelle et singulière (carnets et journaux intimes), privée (correspondance), qui a ses normes, ses règles, ses codes, vers d'autres pratiques (conservation, édition) encore plus encadrées, entraîne une profonde transformation des opérateurs, des contextes de lecture et des usagers et donc de la totalité du dispositif éditorial au sein duquel l'objet prenait initialement son sens. Les usages " natifs » ont été désactivés, surtout lorsqu'il s'agit de dispositifs de flux et non de stockage (lettre, cours, conférence, article), d'autres ont été activés (notamment par la transformation en livres). Par qui l'ont-ils été, pour qui ? Si tout objet s'inscrit dans un usage ou une pratique et inscrit donc en lui cette pratique et cet usage, quels nouveaux usages s'inscrivent dans les nouveaux dispositifs mis en œuvre ? Et que deviennent la trace et la présence fantomatique de l'usage premier ? La lettre reste une lettre, mais elle ne s'inscrit plus dans une pratique de correspondance. L'article de presse reste un article, mais il s'est désancré de la page du journal, il a rejoint, au sein d'un recueil, d'autres articles, antérieurs ou postérieurs, du même auteur ou d'un autre, il n'est plus en résonance avec son actualité ou avec la voix collective à laquelle il participait pour créer une ligne éditoriale et fédérer une communauté de lecteurs.

Loin d'une table rase des pratiques éditoriales du passé, le numérique amplifie donc, comme cela a pu être relevé par ailleurs (Boullier 2016), ces enjeux d'extraction, de décontextualisation, de circulation et de réception... Si l'on peut parler de "remédiation " (Bolter et Grusin 200o), dans la mesure où les nouveaux espaces médiatiques conservent la mémoire de ceux auxquels ils succèdent, on préfèrera pour évoquer l'activité qui nous intéresse ici le terme de "remédiatisation ", au sens où le texte - c'est-à-dire les énoncés et éventuellement leur "disposition " (Dionne 2008), plus rarement leur inscription typographique - migre d'un dispositif vers un autre; le texte se métamorphose en se "remédiatisant ${ }^{8}$ ". On peut dès lors se demander quelle place accorder dans le processus de numérisation à cette mémoire des usages et des différentes strates de médiatisation qui leur sont associées. Dans quelle mesure l'usage "natif » continue-t-il à hanter les nouveaux usages ? C'est en décortiquant les trois vies des écrits de presse de François Mauriac que nous proposons désormais d'esquisser quelques éléments de réponse à cette question.

Cette réflexion s'inscrit dans un programme de numérisation, Mauriac en ligne, porté par le Centre de recherche sur François Mauriac (EA TELEM-UBM) grâce au soutien de la région Nouvelle-Aquitaine. Ce programme a pour ambition de mettre à la disposition de publics variés la 
totalité des textes publiés par François Mauriac dans la presse entre 1905 et 1947. Nous ne chercherons pas ici à retracer l'origine et les différentes étapes de développement de ce projet, mais plutôt à formuler quelques observations sur ces questions de dispositifs éditoriaux à partir de notre propre expérience de terrain. Ce retour sur nos pratiques nous permet de développer une double réflexivité sur les savoirs que nous contribuons ainsi à produire, en même temps que sur les modalités de leur écriture et de leur médiation/médiatisation.

\section{De la presse au livre, une remédiatisation par l'intime}

Les 924 textes aujourd'hui en ligne ${ }^{9}$ ont tous en commun d'avoir été conçus et exploités pour et dans un dispositif éditorial spécifique, ou plutôt dans une modalité de diffusion régulière intégrant des dispositifs éditoriaux primaires très variés (journal quotidien, hebdomadaire, mensuel, parfois illustré, revue, etc.) qui relèvent d'une même " famille ", la presse. Ce sont donc des textes à usage public qui ont accompli en leur temps la fonction pour laquelle ils avaient été conçus : renseigner et débattre sur l'actualité. Numériser et mettre en ligne cette production journalistique conduit à extraire des textes d'autres objets textuels qui ont contribué au fonctionnement d'un dispositif médiatique aujourd'hui totalement désactivé.

Mais avant même cette transformation numérique, une grande partie d'entre eux (467 sur 970) avait déjà fait l'objet d'une extraction et d'une migration vers un autre dispositif éditorial, le livre. À partir des années 1930, François Mauriac a repris en effet lui-même en volumes 354 de ces textes (dont 267 dans les quatre premiers volumes de son Journal), 113 étant par la suite repris dans des recueils publiés après la mort de $\mathrm{Mau}-$ riac, Mauriac avant Mauriac ${ }^{10}$, Mauriac sous l'Occupation, etc. Cette première remédiatisation imprimée ouvre donc un certain nombre de questions qui peuvent éclairer le travail de numérisation qui nous occupe aujourd'hui. Suivre le parcours de quelques articles précis nous permet d'évoquer quelques-unes de ces questions.

Lorsque Mauriac publie chez Grasset en 1934 son Journal, il a déjà pratiqué la reprise en livre de textes publiés dans la presse ${ }^{11}$. Mais il s'agit là de la première pierre d'une partie importante et remarquée de son œuvre. Elle sera en effet suivie de quatre autres volumes du Journal (1934, 1937, 1940, 1950), puis de deux autres entreprises d'envergure, Mémoires intérieurs (1959) et Nouveaux Mémoires intérieurs (1965) et, enfin, des cinq volumes du Bloc-notes (1958, 1961, 1968, 1970, 1971). Par ces nombreuses reprises " autographes ", il s'inscrit lui-même dans une pratique largement stabilisée et intégrée de longue date dans les usages complémentaires des supports journaux et livres, auxquels a notamment recouru la communication littéraire tout au long du XIX ${ }^{\mathrm{e}}$ siècle.

Sans approfondir une analyse qui demanderait un travail d'investigation et de commentaire beaucoup plus poussé, contentons-nous de quelques observations. Dans ce premier volume du Journal, dont la publication (4 avril 1934) suit de très près celle de la dernière chronique retenue (15 novembre 1933), Mauriac retient environ la moitié de la soixantaine de textes qu'il publie dans la presse au cours des années 1932 
et 1933. Le travail mis en œuvre par l'écrivain et son éditeur correspond aux opérations inhérentes à ce type de "remédiatisation " : " mise en texte », puis " mise en livre », il est donc double.

La " mise en texte " correspond au travail de sélection des textes potentiellement " reprenables " effectué par François Mauriac lui-même (sans que l'on connaisse ses critères). Il les dispose, selon une logique qui n'est ni explicite ni explicitée (l'ordre retenu est en partie thématique mais sans véritables règles d'organisation apparentes). Il réécrit ou recompose certains textes, rassemblant par exemple trois articles consacrés à Gide sous un titre commun, "Journal de Gide ", mais il intervient peu sur le contenu et par la suite reprendra ses articles avec le minimum de corrections. Choix, disposition, corrections et éventuellement modifications des titres des articles retenus relèvent donc du premier niveau d'intervention.

Second niveau d'intervention, la « mise en livre ». Ici, le choix du titre, Journal, a évidemment une grande importance, puisque, jouant sur le mot, il renvoie non seulement à l'origine journalistique de ces textes origine rappelée dans l'" avertissement ", lorsqu'il évoque un " recueil d'articles ", mais gommée ensuite par l'absence d'indication des sources des textes -, mais aussi bien sûr à la tradition du journal intime, transformée dans ce même " avertissement " en " journal à demi-intime ". Cette impression est renforcée par le titre du premier texte repris, "Le premier de l'an ", qui donne en effet au lecteur le sentiment d'entrer dans une publication qui relève de la chronique. Par la suite, notamment dans les reprises en volume de ses «bloc-notes ", Mauriac jouera de nouveau des ambiguïtés de ce double dispositif. Extraits de la dernière page de L'Express (ou de La Table ronde pour les premiers), ils ne sont plus disposés en blocs de chroniques (La Table ronde, 6 décembre 1952, etc.) mais en blocs de fragments datés ("Samedi 25 octobre 1952 ", "Lundi 27 octobre 1952 ", etc.). Le lecteur a ainsi le sentiment de lire non une reprise de chroniques mais les pages d'un journal personnel, ou plutôt il peut penser que les chroniques elles-mêmes étaient une première " médiatisation " de textes privés qui auraient eu leur propre espace de déploiement dans un cahier ou des carnets personnels. Le livre, enjambant l'état second qui aurait été celui de la revue, reviendrait alors à l'état premier : pur effet produit par le travail de reprise!

Cette mise en livre mobilise ainsi les différents "seuils " précisément identifiés par Gérard Genette : titre, épigraphe, avertissement, quatrième de couverture, mais également table des matières, voire index pour les volumes des Bloc-notes ou des Mémoires intérieurs. Ces « seuils ", comme l'ensemble des choix de mise en livre, ne délimitent pas seulement un espace, ils constituent les éléments d'un dispositif éditorial, c'est-à-dire d'un agencement conçu en vue d'une pratique de lecture. In fine, ils préparent, encadrent, facilitent, orientent la réception de ces textes. Ils participent aussi de l'étape ultime de métamorphose d'une production médiatique en production livresque ou " littéraire ", l'étape de la «mise en œuvre ». À partir d'une collection de textes indépendants et dispersés, François Mauriac construit une œuvre qui s’intitule Journal et prendra sa place dans sa bibliographie, s'intégrera dans ses Euvres complètes. Ce que ne pourra jamais réaliser une reprise " allographe ", 
celle d'un tiers, contemporain ou éloigné dans le temps, le geste auctorial l'accomplit : il crée une œuvre originale faite de textes qui ne le sont pas.

Ces quelques remarques esquissent les principaux points d'attention que sollicite l'observation de la migration d'un dispositif de départ, en l'occurrence le journal, vers un dispositif d'arrivée, le livre. Cette migration a un impact direct sur l'usage et l'usager, la lecture et le lecteur ; et si ce dernier amortit aisément cet impact, tant il dispose des codes de consultation de l'un comme de l'autre, il est indéniable que la réception du texte en est bien différente. Décontextualisée et " remédiatisée " au sein d'un nouveau dispositif éditorial riche de sens (nouveaux titres et nouvelles associations de textes), l'œuvre journalistique de François Mauriac semble ici relever davantage du journal intime, genre littéraire en soi, que d'une réaction à chaud à l'actualité du monde...

Un exemple plus précis peut nous permettre d'observer plus finement les "protocoles de lecture " (Chartier et Paire 2003, 80) ainsi déposés dans les supports médiatiques. Le 3 décembre 1932, Mauriac publie en " une " de L'Écho de Paris une chronique intitulée "Un soir, Greta Garbo... ${ }^{12}{ }^{\star}$ dans laquelle il rêve que la star pousse la porte de son cabinet de travail pour se confier à lui. Il propose une sorte d'interview imaginaire, qui se démarque des entretiens ou des confidences que les journalistes tentent d'arracher à une vedette très difficile à aborder. Depuis le 2 juillet, Mauriac a débuté avec ce très conservateur Écho de Paris ${ }^{13}$, dirigé par Henry Simond, une collaboration qui marque sa véritable entrée en journalisme. Il y côtoie, en une, Gérard Bauër, Jean-Louis Vaudoyer, les frères Tharaud, André Maurois, Henry Bordeaux, Franc-Nohain ou Paul Morand. Jusqu’à son entrée au Figaro de Pierre Brisson, en juin 1934, il y publiera, le samedi, deux fois par mois, quarante-deux chroniques, presque toutes reprises dans le premier volume du Journal. La forme utilisée, une chronique régulière composée ici en forme de pseudo-interview, le sujet surtout, s’intègrent parfaitement dans le cadre de lecture auquel est accoutumé le lecteur du journal. Celui-ci trouve dans chaque numéro de son quotidien une chronique de deux colonnes, à gauche en une, qui côtoient les autres colonnes (sept au total) consacrées à l'actualité politique, nationale ou internationale, ou à de spectaculaires faits divers. La chronique en question s'inscrit de plus dans une actualité dont le lecteur est parfaitement informé : Garbo est arrivée en France pour la sortie d'un film, Mata Hari, mais toute la presse la traque, la cherche, car elle fuit les journalistes et se serait même déguisée pour décourager leurs poursuites. Le lecteur ne peut donc que sourire à la lecture de ce texte : alors que tout Paris se demande « Où est Greta Garbo ? ", il la découvre poussant la porte du cabinet de travail de Mauriac. Quel scoop ! Le contenu même de l'article reprend ce dont se nourrit toute la presse de l'époque ${ }^{14}$, pour développer, sur cette base anecdotique et légère, une véritable méditation spirituelle. Or, l'extraction du texte réduit à ses énoncés, avec quelques légères modifications, corrections ou suppressions, sans conservation de la typographie et de l'espace de consultation partagé avec d'autres textes d'auteurs et de teneur très différents, son insertion enfin dans un autre espace de consultation, celui du livre, bouleversent les modalités de consultation et de réception du texte. Ce geste l'arrache du même coup au contexte de lecture qui contribue aussi à lui donner sinon du sens du moins un sens, pour l'inscrire dans un mode de 
communication différable, dans lequel le rapport à une actualité identifiée ne se pose plus avec la même acuité. Le lecteur le découvre au sein du Journal encadré par des textes beaucoup plus sombres ${ }^{15}$, des textes qui donnent naissance par leur assemblage à une œuvre neuve au sein de laquelle ils vont trouver désormais leur place et leur sens - un ensemble de textes où François Mauriac évoque le théâtre et les actrices - et au sein duquel le lecteur, dans une pratique personnelle et là aussi intimiste, va se déplacer à sa guise (grâce à la table des matières) et à son rythme, à la découverte de ce recueil de pensées intimes.

Ces remarques nous permettent donc de souligner combien l'article de presse, texte par essence autonome et public mais partie prenante d'une publication plurielle, se métamorphose, en se "remédiatisant " dans l'objet livre en une partie d'un tout plus large. On peut dès lors se demander s'il s'agit toujours du même texte. Répondre par l'affirmative à cette question reviendrait à nier l'importance du dispositif éditorial dans le processus de réception et donc de signification, tout comme la part de création, de réinvention, qu'il y a dans le geste de la reprise. Le déplacement, la migration d'un dispositif éditorial vers un autre produit nécessairement une altération, une métamorphose.

\section{Et de la presse au Web, une remédiatisation par dissémination}

La publication de presse s'inscrit donc dans une chaîne de production et de communication qui mobilise des compétences complémentaires au sein d'un dispositif éditorial à la fois industriel (économique et technique), rédactionnel (rubriquage, genres, contenus, styles), politique et social, voire symbolique (valeur sociale et positionnement de la publication). La publication en livre s'inscrit quant à elle dans une tout autre logique, pas seulement en termes de chaîne de production, mais aussi de processus de signification : valorisation d'une parole singulière, décontextualisation de textes, valorisation de l'objet et conservation, etc. Or, un projet de numérisation et de mise en ligne d'objets textuels au sein d'un nouveau dispositif éditorial, qui, pour paraphraser Pierre-Yves Buard $(2015,30)$, consiste à fabriquer une version numérique de ces textes mis en forme et directement utilisables par le lecteur, pose les mêmes questions car il relève en fin de compte de la même logique d'extraction et de migration vers un nouveau dispositif. Il induit, lui aussi, une modification de l'environnement de production et de consultation et s'inscrit dans une autre pratique, celle de la consultation d'une base de données. Autrement dit, lui aussi métamorphose totalement le texte en le " remédiatisant ". Le travail mené par l'équipe mobilisée sur le projet Mauriac en ligne et les choix effectués permettent de cerner les enjeux de cette « remédiatisation".

La construction de ce nouvel espace de connaissances passe en premier lieu par la reproduction numérique des textes journalistiques de François Mauriac. Mais cette reproduction n'est évidemment pas neutre, elle est affaire là encore de choix et de contextes, non seulement scientifiques et techniques, mais aussi politiques. Si parmi les nombreux projets associés au sein du consortium CAHIER, un grand nombre se consacrent avant tout à l'édition électronique de manuscrits anciens et 
modernes ${ }^{16}$, seul le projet Édition des journaux d'Alexandre Dumas travaille un corpus de presse ${ }^{17}$. Or, la confrontation du dispositif de "remédiatisation " de ces textes avec celui du site Mauriac en ligne montre combien les solutions adoptées divergent : outils développés ad hoc pour l'un, adaptation d'un outil générique pour l'autre ; présentation in extenso des journaux pour l'un, extraction des articles " mauriaciens " pour l'autre ; recherche par index pour l'un, recherche plein texte pour l'autre, etc. Chacun de ces projets répond certes parfaitement aux règles de la discipline scientifique (retour au document original, présentation des sources) et à celles de la diffusion numérique (structuration des données, respect des formats internationaux de description), mais les objectifs de lecture définis par les équipes engagées dans ces projets ont évidemment contribué à l'élaboration de dispositifs éditoriaux bien différents.

Le projet Mauriac en ligne a ainsi débuté dès 2011 avec l'inventaire et le récolement, à l'aide d'outils bibliographiques, des 3 ooo textes de presse rédigés par François Mauriac entre 1905 et 1970. Cette étape de localisation franchie, et après une première expérimentation d'édition numérique réalisée avec l'université d'Exeter au format XML-TEI ${ }^{18}$, divers fonds ont été sollicités à partir de 2014 pour compléter la collection réunie par le Service commun de documentation de l'université Bordeaux-Montaigne et obtenir ainsi au format numérique tous les articles parus, à quelques exceptions près, entre 1905 et 1947. Pour des questions juridiques, seule cette première production journalistique peut en effet pour l'instant être diffusée en libre accès. Repérage, collecte, récolement et numérisation effectués, les articles ont ensuite été structurés au sein d'une base de données publique construite selon quatre grands principes éditoriaux : 1) l'exhaustivité, en recherchant l'intégralité des articles publiés par François Mauriac ; 2) le respect de la spécificité de l'ensemble des textes, hétérogène et fragmenté, issu d'une production périodique qui a souvent fait l'objet de reprises des mains mêmes de l'écrivain par la suite ; 3) un accès libre, ouvert et gratuit ; 4) une diffusion enrichie à partir d'une réflexion sur les usages et les lecteurs visés. Si nous ne reviendrons pas sur les choix techniques effectués, déjà interrogés ailleurs (Bideran 2016), signalons que c'est par un détournement des fonctionnalités du CMS Omeka (exploitation du plugin Scripto et extension des métadonnées) que l'édition numérique proposée permet de conjuguer l'exposition des images (fac-similés des articles de François Mauriac océrisés) avec la publication des textes (articles transcrits).

Cette «troisième vie " des textes de presse, nous l'avons donc conçue non pas en rupture, mais dans le prolongement des deux premières. Certes, contrairement à la reprise en livre effectuée par l'auteur luimême, elle ne fait pas " œuvre ", pas plus d'ailleurs que les reprises posthumes de textes laissés pour compte par Mauriac et effectuées par des spécialistes. Mais elle va plus loin ; elle "dés-œuvre ", car elle " délivre $^{19}$ ». En revenant à l'état de dispersion, elle permet de retrouver la logique de production originale, celle d'une production de fragments en flux continu. Le choix éditorial consistant par exemple à proposer une version image en complément d'une version texte pour chacun des articles vise ainsi à restituer la matérialité du texte de presse. Alors que les reprises en livre avaient uniformisé ces objets textuels en neutralisant 
leur matérialité, la numérisation de l'ensemble de la production journalistique, par l'identification du périodique, de la date de parution et du contexte éditorial, refragmente ce qui avait été rassemblé.

Au début de l'année 2019, la base de données réunissait ainsi plus de 920 textes - soit près de $95 \%$ des textes de cette première période, une petite vingtaine de textes restant à ce jour introuvables -, transcrits et décrits au format Dublin Core enrichi, également accessibles depuis le portail Isidore qui moissonne les métadonnées grâce au protocole d'échange OAI-PMH. Ce chantier, qui s'est construit progressivement et selon une démarche pragmatique, interdisciplinaire et décloisonnée, dépasse ce premier travail de reproduction des textes. Affranchis de leur milieu d'origine, ces derniers sont en effet reconfigurés par un jeu de fragmentation et de structuration qui autorise l'élaboration de nouvelles "fictions" (Merzeau 2006) autour de l'œuvre journalistique mauriacienne. Celles-ci dépendent des contextes de lecture de ces objets textuels, que nous pouvons formaliser à partir d'études qualitatives menées auprès des publics en ligne du patrimoine numérisé (Chevallier, Rioust et Bouvier-Ajam 2011) ; aux pratiques de recherche sémantique et de lecture exhaustive élaborées par les chercheurs semblent ainsi répondre des pratiques de lecture par vagabondage et thématique des passionnés et des publics scolaires.

Du côté des usages de lecture experte, de nouvelles questions interprétatives peuvent être posées. Une recherche en cours liée à la récente réédition de L'Élève Gilles, ouvrage publié en 1912 par André Lafon et préfacé par François Mauriac, permet d'illustrer rapidement ce potentiel. L'interrogation de la base de données fait ainsi remonter 29 textes où Mauriac évoque son amitié avec cet écrivain bordelais décédé en 1915 durant la Première Guerre mondiale, bien loin des 3 textes traditionnellement cités par la critique mauriacienne ${ }^{20}$. Pour anecdotique que soit cet exemple, il permet de souligner combien le dispositif médiatique envisagé, par isolement, rapprochement ou confrontation, revalorise le statut non seulement de ces textes, mais aussi plus globalement du corpus et de l'œuvre journalistique mauriacienne en établissant de nouveaux réseaux entre des éléments autrefois distincts. Car la base de données ne stocke pas "des artefacts muets" (Gefen 2015), mais des écrits d'écran qui supposent écriture, lecture et manipulation. De fait, se pose la question des cheminements, aléatoires ou encadrés, des combinatoires diverses, par période, par thèmes, par genre d'écriture, etc., possibles au sein de ce corpus. Désormais décomposés dans de nouvelles unités informationnelles, les textes consultables via la base sont devenus des données lisibles grâce aux métadonnées associées.

Sont donc ici rassemblés des signes de nature différente : des mots (les écrits retranscrits de François Mauriac), des images (les fac-similés numériques de ces articles) et des métadonnées, c'est-à-dire des «hypermots" (Jeanneret 2001) qui permettent de circuler au sein du corpus. Ces différents accès sont assurés par des outils de structuration qui relèvent d'ailleurs plus d'une certaine folksonomie "grand public » que du thésaurus spécialisé. Au nuage de mots-clés, qui construit peu à peu un univers sémantique et ontologique typiquement mauriacien mêlant noms propres (André Gide, Jacques Rivière, Georges Duhamel, etc.), noms communs (" grâce ", " amour ", " enfance ", etc.), locutions ("acteurs et actrices ", " littérature engagée ", etc.) et adjectifs (" mufle », 
" immonde ", « débauché », etc.), répond ainsi une cartographie qui permet de visualiser une série de textes évoquant les sites et paysages aquitains, de matérialiser dans l'univers numérique les liens entre l'œuvre de François Mauriac et le territoire aquitain. D’autres parcours enfin sont suggérés au sein de collections thématiques permettant de proposer à la lecture des regroupements de textes parfois inédits ("Le Front populaire », « La guerre d'Espagne », etc.).

Or, l’obligation de générer des métadonnées pour décrire les textes et permettre leur accessibilité suppose un travail de longue haleine de relecture des textes et s'accompagne, de la part de l'équipe engagée dans un tel projet, d'une adaptation de la pensée à l'outil, et inversement. Car cette profusion de signes et d'accès entre aussi en tension avec le formatage du CMS retenu - l'architexte pour paraphraser Genette et à sa suite Jeanneret -, que nous tentons de contourner dans une bataille permanente entre solution technique facile à prendre en main et transcription d'une complexité culturelle inhérente à tout objet textuel. La solution logicielle adoptée - un outil de structuration et de publicisation de bibliothèque numérique qui exige notamment le recours aux fac-similés numériques et permet donc de conserver la mémoire de l'objet textuel premier - s'avère à la longue peu adaptée aux multiples interprétations possibles de nos textes. Le détournement de la fonctionnalité « collection ", initialement dédiée à une structuration par typologie d'objets (manuscrits, photographies, imprimés par exemple), pour créer les parcours thématiques que nous venons d'évoquer nous a ainsi amenés à une impasse, un même texte pouvant chez François Mauriac aborder la guerre d'Espagne et le Front populaire.

Dépasser cet encadrement du dispositif éditorial nous a conduits à multiplier les présences en ligne de nos objets textuels en élaborant, à côté de notre base de données, un blog sur la plateforme scientifique $H y$ pothèses. Celui-ci offre un second contexte de lecture numérique qui nous permet de développer nos réflexions, de confronter nos objets textuels tout en les liant à d'autres ressources numériques repérées sur le Web, à l'image du billet sur la guerre d'Espagne qui permet de visualiser une archive de l'INA au cours de laquelle François Mauriac revient sur son engagement. Cet autre espace numérique se sert donc du premier dispositif (la base de données) comme d'une réserve permettant d'accéder aux "sources primaires et [...] autorités qui fiabilisent l'information " (Laborderie 2016). Ce troisième "formatage " de nos objets textuels au sein de dispositifs éditoriaux numériques agit en fin de compte sur la nature même du corpus, désormais multimédia et ouvert à d'autres ressources que nos seuls textes, qui s'interconnectent avec d'autres documents. Au-delà des gestes techniques et documentaires, mettre en ligne des corpus d'auteurs, c'est en définitive se lancer dans une opération d'" éditorialisation " (Vitali Rosati 2016) qui permet d'étendre les modes de présence en ligne des objets textuels par une ouverture de l'espace de lecture (plusieurs plateformes) et une multiplication des acteurs concernés (chercheurs, bibliothécaires, lecteurs, etc.). 


\section{Conclusion}

Au terme de cette réflexion et en guise de conclusion, nous nous proposons d'esquisser rapidement les enjeux qui accompagnent de tels programmes d'édition numérique. Ici, la responsabilité de l'opérateur dans la mise en œuvre du dispositif éditorial est essentielle. Au lien auteur/lecteur se superpose en effet un lien opérateur/usager. Relèvent du premier niveau les réflexions sur la lecture et la maîtrise du sens du texte (études de réception et travaux de Barthes, Eco, Genette, etc.). Du second niveau, la maîtrise du fonctionnement du dispositif qui concerne le courant des humanités numériques. Comme pour le premier, on peut distinguer au sein des analyses deux pôles ou deux tendances. Pour les uns, le dispositif maîtrise l'usage, il le prévoit, il l'encadre, voire le verrouille. Pour d'autres analystes, l'usager s'émancipe du projet porté par l'opérateur ; il se l'approprie, voire le détourne. À la fois acte de résistance, voire d'émancipation, et activité créatrice, l'usage se définit en termes de bricolage, de braconnage (de Certeau), voire de piratage.

Dans le premier cas, l'usage et l'usager sont inscrits dans le dispositif, sous forme de "scripts "- selon Madeleine Akrich (1993), toute opération est envisagée en fonction d'un usage virtuel, plus ou moins explicitement défini et qui s'actualise à l'usage - qui se déclinent en attentes, compétences, comportements, performances. Dans le second cas, ils s'inscrivent eux-mêmes dans la chaîne de fonctionnement et peuvent en inverser le cours et la hiérarchie. Une approche qui vise la mise à disposition des données, exigence minimale mais fondamentale pour les projets associés au sein de CAHIER, est davantage du côté du second pôle que du premier.

Mais la diversité des usages des dispositifs numériques est-elle profondément liée à la nature des corpus numérisés ou dépend-elle uniquement des modalités de remédiatisaton choisies ? La plateforme $E M A N^{21}$, réalisée elle aussi à partir du CMS Omeka et réunissant à partir d'une même architecture de nombreux et complexes corpus, permet de souligner l'importance de la typologie du corpus dans l'appropriation par les usagers - ici scientifiques - du dispositif d'édition. La stratification des usages et des modalités de médiatisation peut être source de conflits car toute médiatisation ou médiation suppose un choix orienté et donc contestable. Conflit entre conservation et diffusion, entre différents supports de médiatisation, entre exigence scientifique (qui tire « vers le haut») et vulgarisation (qui tirerait "vers le bas»).

La recherche d'une voie médiane est-elle la solution? Est-elle une utopie ? La multiplicité des usages demande en tout cas à être inscrite au sein du dispositif. Les activités d'indexation et de transcription collaboratives (crowdsourcing) sur des stéréoscopies anciennes ${ }^{22}$ ou sur des carnets d'ethnologues ${ }^{23}$ montrent en tout cas que le dispositif élaboré façonne dans ces cas-là des communautés d'usagers amateurs dont les actions sont riches de sens pour le scientifique, mais aussi largement encadrées par le corpus et par le système de remédiatisation. L'identification de ces usages semble donc un prérequis essentiel lors de l'élaboration d'un dispositif : scientifique, pédagogique (envisagé souvent au niveau du second degré, le supérieur apparaissant peu), culturel... et d'autres sans doute qui restent à explorer et définir. La mise en œuvre de 
corpus numériques façonne dans tous les cas de nouveaux objets de recherche, ainsi que des méthodologies toujours en cours de questionnement, et amène nécessairement à réfléchir sur l'extension des régimes d'éditorialisation, ces derniers s'inscrivant par ailleurs dans les nombreuses injonctions qui déterminent aujourd'hui le financement de la recherche (injonction à la numérisation, à la participation, à la valorisation, etc.).

\section{Bibliographie}

Akrich, Madeleine. 1993. «Technique et médiation». Réseaux. Communication, technologie, société 11 (6o) : 87-98. https://doi.org/10.3406/reso.1993.2368.

Bideran (de), Jessica. 2016. « Du fragment daté au corpus patrimonialisé : numérisation et muséalisation de l'article de presse mauriacien ". Études digitales 1 (1) : 125-142.

Bolter, Jay David et Richard Arthur Grusin. 200o. Remediation : Understanding New Media. Cambridge : The MIT Press.

Bonaccorsi, Julia. 2015. "Quelle réflexivité médiatique pour les humanités numériques? La numérisation des manuscrits littéraires ». Les Enjeux de l'information et de la communication $2: 83-97$.

Boulaire, Cécile et Roméo Carabelli. 2017. « Du digital naive au bricoleur numérique : les images et le logiciel Omeka ". Dans Expérimenter les humanités numériques. Des outils individuels aux projets collectifs. Édité par Étienne Cavalié, Frédéric Clavert, Olivier Legendre et Dana Martin, 81-103. Montréal : Presses de l'université de Montréal. http://www.parcoursnumeriques-pum.ca/du-digital-naive-au-bricoleur-numerique.

Boullier, Dominique. 2016. Sociologie du numérique. Paris : Armand Colin.

Buard, Pierre-Yves. 2015. "Modélisation des sources anciennes et édition numérique ». Thèse de doctorat en informatique et applications, université de Caen. https://hal.archives-ouvertes.fr/tel-01279385.

Chartier, Roger et Alain Paire, éd. 2003. Pratiques de la lecture. Paris : Payot.

Chevallier, Philippe, Laure Rioust et Laurent Bouvier-Ajam. 2011. « La consultation de manuscrits en ligne ». Bulletin des bibliothèques de France 5 : 7 .

Davallon, Jean. 2006. Le Don du patrimoine : une approche communicationnelle de la patrimonialisation. Paris : Lavoisier.

Dionne, Ugo. 2008. La Voie aux chapitres : poétique de la disposition romanesque. Paris : Seuil.

Eberle-Sinatra, Michael et Marcello Vitali Rosati, éd. 2014. Pratiques de l'édition numérique. Montréal : Presses de l'université de Montréal.

Gefen, Alexandre. 2015. «Les enjeux épistémologiques des humanités numériques ». Socio 4 (avril) : 61-74. https://doi.org/10.4000/socio.1296.

Genêt, Pascal. 2019. "Énonciation éditoriale ». Socius. http://ressources-socius.info/index.php/lexique/21-lexique/190-enonciation-editoriale.

Idmhand, Fatiha, Claire Riffard et Richard Walter. 2017. "L'édition électronique de manuscrits modernes ». Dans Expérimenter les humanités numériques. Des outils individuels aux projets collectifs. Édité par Étienne Cavalié, Frédéric Clavert, Olivier Legendre et Dana Martin, 105-123. Montréal : Presses de l'université de Montréal. http://www.parcoursnumeriques-pum.ca/l-edition-electronique-de-manuscrits-modernes.

Jeanneret, Yves. 2001. «Les politiques de l'invisible». Document numérique 5 (1) : 155-180. h ttps://www.cairn.info/revue-document-numerique-2001-1-page-155.htm.

Jeanneret, Yves. 2007. «Usages de l'usage, figures de la médiatisation ». Communication \& langages 151 (1) : 3-19. https://doi.org/10.3406/colan.2007.4629.

Jeanneret, Yves et Emmanuël Souchier. 2005. "Lénonciation éditoriale dans les écrits d'écran ". Communication \& langages 145 (1) : 3-15. https://doi.org/10.3406/colan.2005.3351.

Jouët, Josiane. 200o. "Retour critique sur la sociologie des usages ». Réseaux. Communication, technologie, société 18 (100) : 487-521. https://doi.org/10.3406/reso.2000.2235. 
Laborderie, Arnaud. 2016. "Éditorialisation des bibliothèques numériques : le cas des Essentiels de Gallica ». Dans Documents et dispositifs informationnels à l'ère post-numérique. Actes $d u 18^{e}$ colloque international sur le document électronique. Édité par Céline Paganelli, Stéphane Chaudiron et Khaldoun Zreik. Paris : Europia. https://hal.archives-ouvertes.$\mathrm{fr} / \mathrm{hal}-01239425$.

Le Deuff, Olivier. 2016. " Anatomie et nouvelle organologie de l'édition ouverte ». Revue française des sciences de l'information et de la communication 8 (janvier). https://doi.org/10. 4000/rfsic.1871.

Le Marec, Joëlle. 2002. «Ce que le "terrain” fait aux concepts : vers une théorie des composites ». Habilitation à diriger des recherches, Paris : université Paris-Diderot.

Merzeau, Louise. 2006. «Malraux metteur en page ». Dans Les Écrits sur l'art d'André Malraux. Édité par Jean-Yves Guérin et Julien Dieudonné, 64-79. Paris : Presses Sorbonne Nouvelle.

Pédauque, Roger T. et Niels Windfeld Lund. 2007. La Redocumentarisation du monde. Toulouse : Cépaduès Éditions.

Peeters, Hugues et Philippe Charlier. 1999. «Introduction. Contributions à une théorie du dispositif ». Hermès 25 : 15-23. https://doi.org/10.4267/2042/14969.

Thérenty, Marie-Ève. 2013. La Littérature au quotidien : poétiques journalistiques au XIX ${ }^{e}$ siècle. Paris : Seuil.

Vitali Rosati, Marcello. 2016. « Qu'est-ce que l'éditorialisation ?» Sens public (mars). http:// www.sens-public.org/articlel184.html.

\section{Notes}

1 L'usage qui sera fait ici de ce terme s'inscrit dans la définition donnée par Peeters et Charlier (1999). Le dispositif tel qu'on le comprend ici est donc avant tout " technique "; c'est celui qu'ils définissent comme une « logique de moyens mis en œuvre en vue d'une fin ». Il suppose une visée, une stratégie, un fonctionnement suscitant (sans les déterminer) des procédures et des usages, ceux que l'on peut identifier dans un dispositif éditorial, en l'occurrence ceux de la presse, du livre et du numérique.

2 Notamment lors de l'atelier annuel 2017 du consortium, qui eut lieu du 27 au 30 juin à l'invitation du projet Mauriac en ligne, au Centre François Mauriac de Malagar et à la Maison des sciences de l'homme d'Aquitaine, cf. http://cahier.hypotheses.org/3235.

3 Le projet Mauriac en ligne s'inscrit ainsi aujourd'hui dans le cadre d'un nouveau programme de recherche réunissant des équipes d'universitaires néo-aquitains et travaillant sur différents corpus d'auteurs : $e$-CANA, Étudier, numériser et exploiter les corpus d'auteurs en Nouvelle-Aquitaine.

4 Nous distinguons ici ces deux modes de présence du texte dans l'espace public, le premier correspondant selon nous au processus de circulation et transmission des messages à travers un support ou un outil de communication, le second se situant du côté de la relation directe qui s'instaure entre l'objet textuel et les usagers, et se positionnant donc du côté de la réception et non de la diffusion, comme c'est le cas de la médiatisation.

5 On pourra toutefois souligner la récente publication en ligne d'un guide (version 1) qu'il a produit à l'issue des travaux du groupe menés entre octobre 2016 et octobre 2018.

6 Bibliothèque publique patrimoniale consacrée à la littérature française de la seconde moitié $\mathrm{du} \mathrm{XIX}^{\mathrm{e}}$ siècle à nos jours. Créée par le couturier Jacques Doucet, léguée à l'Université de Paris en 1929, elle relève depuis 1972 de la chancellerie des universités de Paris.

7 On pourra par exemple se reporter à l'histoire de l'édition savante ou critique, de ses objectifs, de ses pratiques.

8 Pour Jay-David Bolter et Richard Grusin (200o), la « remédiation » désigne la représentation d'un média dans un autre média. Ce terme, soulignant plutôt la présence usuelle d'un média dans l'autre, peut être distingué de "remédiatisation », lequel ne concerne pas la simple visualisation des formes qui font évoquer un autre média, mais plutôt le transfert des données génératrices de l'autre média (ou "générées par l'autre média ») induisant de nouvelles pratiques et de nouveaux cadres de perception et d'action.

9 Les quantités indiquées ici et dans les lignes qui suivent ont pour but de donner un ordre de grandeur ; les chiffres varient selon les critères que l'on retient (nature des textes retenus, prise en compte des reprises, etc.). 
10 Mauriac avant Mauriac, textes retrouvés, présentés et annotés par Jean Touzot, Flammarion, 1977.

11 Dramaturges, publié en 1928 à la Librairie de France reprend une partie des chroniques dramatiques publiées dans La Revue hebdomadaire ou à la NRF.

12 Voir la version originale sur le site Mauriac en ligne : http://mauriac-en-ligne.u-bordeaux-montaigne.fr/items/show/452.

13 Que son directeur définissait ainsi : «Un quotidien dont le lecteur est prêtre, père ou frère de prêtre, et la lectrice une dame en deuil qui a son fils à Saint-Cyr. "

14 Par exemple l'article de Louis Chauvet, «La vedette invisible », paru en page 2 du Figaro du 17 novembre 1932 et accessible en ligne sur Gallica : http:/gallica.bnf.fr/ark:/12148/bpt6k297150h.

15 Celui qui le précède dans le volume s’intitule «Jour des morts » et ceux qui le suivent, « Le noir » et " Anna de Noailles est morte », extraits de L'Écho de Paris ou des Nouvelles littéraires pour le dernier, et respectivement accessibles en ligne sur le site Mauriac en ligne aux adresses http://mauriac-en-ligne.u-bordeaux-montaigne.fr/items/show/450, http:// mauriac-en-ligne.u-bordeaux-montaigne.fr/items/show/465 et http://mauriac-en-ligne.u -bordeaux-montaigne.fr/items/show/533. La confrontation des textes repris en volume avec leur mise en ligne permettra à cet égard de souligner, a minima, les différences de titres de ces articles.

16 Voir la liste des membres : http://cahier.hypotheses.org/membres.

17 http://alexandredumas.org/.

18 Portant sur les années 1936-1937, cette édition numérique est encore accessible en ligne : http://mauriac.ex.ac.uk/.

19 Selon l'heureuse expression de Claire Clivaz et Dominique Vinck, dérivée du titre de leur colloque «Les humanités délivrées " (Lausanne 2013), dont des actes partiels ont été publiés dans Les Cahiers du numérique 3, 10, 2014.

20 Voir ici : https://goo.gl/jgfH5R. Les trois textes cités étaient les suivants : «Examen de conscience sur la tombe d'André Lafon ", publié le 12 janvier 1924 dans La Revue hebdomadaire et repris avec le titre « Examen de conscience " par Mauriac dans différents volumes (http://mauriac-en-ligne.u-bordeaux-montaigne.fr/items/show/726) ; "L'un d'eux : André Lafon ", publié le ${ }^{\mathrm{er}}$ juillet 1916, dans La Revue hebdomadaire (http://mauriac-en-ligne.u-b ordeaux-montaigne.fr/items/show/653) ; "La Correspondance d'André Lafon », publié le 10 août 1918 dans la Revue des jeunes (http://mauriac-en-ligne.u-bordeaux-montaigne.fr/items/show/631).

$21 \mathrm{http}: / /$ www.eman-archives.org/.

22 http://p3dstereo.huma-num.fr/.

23 http://transcrire.huma-num.fr/.

\section{Auteurs}

\section{Philippe Baudorre}

EA 4195 TELEM, université Bordeaux-Montaigne, Pessac, France

Philippe Baudorre est professeur de littérature française à l'université Bordeaux-Montaigne et membre de l'EA 4195 TELEM (Textes, littératures : écritures et modèles). Il travaille plus particulièrement sur les relations entre presse et littérature au $\mathrm{xx}^{\mathrm{e}}$ siècle.

Philippe.Baudorre@u-bordeaux-montaigne.fr

\section{Jessica de Bideran}

EA 4426 MICA, université Bordeaux-Montaigne, Pessac, France

Jessica de Bideran est ingénieure de recherche à l'université Bordeaux-Montaigne et membre de l'EA 4426 MICA (Médiations, informations, communication, arts). En charge notamment du programme Mauriac en ligne, elle interroge, à travers ces différents travaux, les multiples présences numériques des patrimoines.

jessica.de-bideran@u-bordeaux-montaigne.fr 


\section{Droits d'auteur}

(c) (7)

Les contenus de la revue Humanités numériques sont mis à disposition selon les termes de la Licence Creative Commons Attribution 4.0 International. 